\title{
Indian College of Radiology and Imaging (ICRI) Consensus Guidelines for the Early Management of Patients with Acute Ischemic Stroke: Imaging and Intervention
}

\author{
Shrikant R Londhe ${ }^{1}$ Sharath Kumar G G ${ }^{2}$ Shyamkumar N Keshava ${ }^{3} \quad$ (Brig) Chander Mohan ${ }^{4}$
}

1Department of Interventional Neuroradiology, Noble Hospital, Pune, Maharashtra, India

2Department of Diagnostic and Interventional Neuroradiology, Apollo Hospitals, Bangalore, Karnataka, India

${ }^{3}$ Department of Interventional Radiology, Christian Medical College, Ida Scudder Road, Vellore, Tamil Nadu, India

4 Interventional Radiology, ICRI Director, Interventional Radiology, BLK Super Specialty Hospital, Pusa Road, New Delhi, India

\begin{abstract}
Address for correspondence Sharath Kumar G G, Department of Diagnostic and Interventional Neuroradiology, Apollo Hospitals, Bangalore, Karnataka, India (e-mail: drsharathkumargg@gmail.com).
\end{abstract}

Indian J Radiol Imaging 2021;31:400-408.

\begin{abstract}
Keywords

- acute ischemic stroke

- endovascular mechanical thrombectomy

- large vessel occlusion

The medical science has witnessed significant change in the management of acute stroke patients as a result of recent advances in the field of stroke imaging and endovascular mechanical thrombectomy in addition to intravenous thrombolysis and optimization of stroke services in balance with available resources. Despite initial negative trials, we witnessed the publication of five multicenter randomized clinical trials showing superiority of the endovascular approach over standard medical management in patients with large vessel occlusion. The aim of this study is to provide comprehensive set of evidence-based recommendations regarding imaging and endovascular interventions in acute ischemic stroke patients.
\end{abstract}

\section{Introduction}

Acute ischemic stroke (AIS) is a time-sensitive condition. On average, 1.9 million neurons die per minute in the hypoperfused ischemic penumbra that surrounds the core of the infarction. ${ }^{1}$ This sense of urgency should guide all the aspects of the patient evaluation and management. It is critically important that stroke centers, primary and comprehensive, have well-designed imaging and interventional protocols for acute stroke patients that are automatically triggered either on arrival or upon notification of patient transport.

We are in the dawn of a new era for the treatment of cerebrovascular diseases. The beginning of this new road was the development of endovascular therapy (ET). The endovascular era undoubtedly represents a revolutionary paradigm shift to treat cerebral circulation pathology by the inside of the vessel rather than by an open surgical approach. Over the last few years, based on results of multiple randomized controlled trials (RCTs), emergency stroke management has seen big leap in terms of stroke center organization, imaging, and endovascular mechanical thrombectomy (MT) essentially improving the functional outcome of the patients. In this article, we have provided evidence-based recommendations ( - Fig. 1 ) regarding the imaging (Part I) and endovascular MT (Part II) in AIS patients. published online July 27, 2021
DOI https://doi.org/

$10.1055 / \mathrm{s}-0041-1734346$ ISSN 0971-3026 (c) 2021. Indian Radiological Association.

This is an open access article published by Thieme under the terms of the Creative Commons Attribution-NonDerivative-NonCommercial-License, permitting copying and reproduction so long as the original work is given appropriate credit. Contents may not be used for commercial purposes, or adapted, remixed, transformed or built upon. (https://creativecommons.org/licenses/by-nc-nd/4.0/).

Thieme Medical and Scientific Publishers Private Ltd. A-12, Second Floor, Sector -2, NOIDA -201301, India 


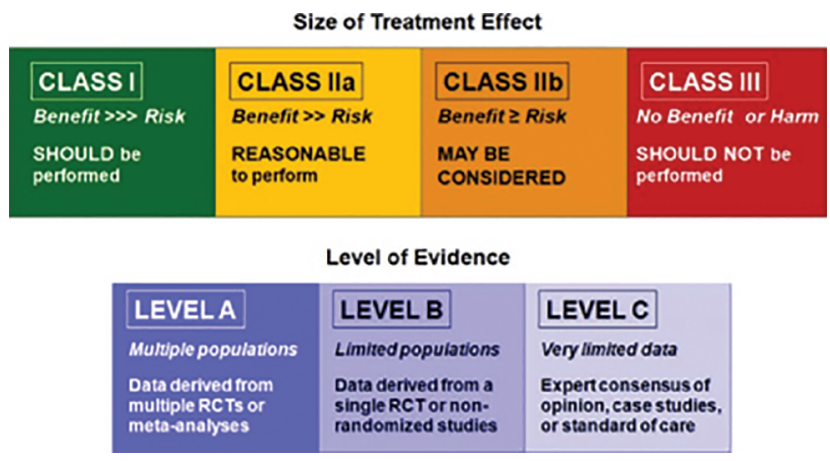

Fig. 1 Class of recommendation and level of evidence to clinical strategies, diagnosis, intervention, or treatment in patient care. RCT, randomized controlled trial.

\section{PART I: Neuroimaging in Acute Ischemic Stroke}

\section{Patient with Suspected Acute Stroke Should Get an Urgent Neuroimaging Evaluation before Initiating Any Specific Treatment (I-A)}

In AIS patients within window period, benefit of intravenous thrombolysis (IVT) is time dependent. ${ }^{1}$ Early initiation of treatment has shown better functional outcome (modified Rankin's scale-mRS) across all age groups and stroke severity. ${ }^{2-4}$ National Institute of Neurological Disorders and Stroke (NINDS) trial considered noncontrast computed tomography (NCCT) brain as the only imaging evaluation needed before IVT administration. ${ }^{5}$ European Cooperative Acute Stroke Study (ECASS) trial also used NCCT brain imaging to detect early signs of acute infarction like sulci effacement, obscuration of deep gray mater, loss of insular cortical hyperdensity, and hyperdense artery sign. ${ }^{6}$ Routine use of magnetic resonance imaging (MRI) brain in hyperacute stroke has certain limitations like safety issues, artifacts in uncooperative patient, and longer time for scan. In comparison to NCCT brain, delay of an average 10 minutes (door to needle time) has been shown whenever MRI is used as primary imaging modality even in well-organized hospital settings.,

Based on available evidence and recommendation, early neuroimaging for any suspected acute stroke should be initiated without any delay for rapid diagnosis of ischemic or hemorrhagic stroke subtypes.

\section{Hospital Administration System of Stroke Pathway Should Be Established to Facilitate Quick Stroke Imaging Pathway Especially in Acute Stroke Patient Who are Potential Candidates for Intravenous Thrombolysis/Mechanical Thrombectomy (I-A)} Effective stroke pathway for all the patients of suspected acute stroke should be established to achieve rapid management that leads to better clinical outcome. Multiple studies have shown the importance of establishing in hospital stroke pathway and formation of dedicated stroke team., ${ }^{2,49}$ Stroke audit of all the patients should try to achieve desired time deadline as benefit of any interventions in AIS declines over the time. ${ }^{10}$ Effective training standards of stroke paramedics and emergency department team can help achieving the smooth functioning of stroke pathway with multiple channels working toward common goal. ${ }^{11}$

Acute stroke team with hospital administration should target specific door to needle (for IVT) and door to puncture time (for MT) as per international standards. Stroke protocol imaging should always be directed to give necessary information in shortest time with available imaging modalities.

\section{Noncontrast CT Brain Is Effective to Exclude Intracerebral Hemorrhage (ICH) before Intravenous Thrombolysis Administration in Acute Stroke Patients of Onset Less Than 4.5 Hours (I-A)}

Noncontrast CT has highest sensitivity to identify $\mathrm{ICH} .^{8}$ In addition to this, certain other contraindications like acute subdural hemorrhage and neoplasm/space-occupying lesion can be easily ruled out with CT before starting IVT. ${ }^{12}$ MRI-based protocol also has shown equal sensitivity to detect ICH; few other factors like cost, patient-related artifacts, and scan time play role in selection of one over the other. Some contraindications for MRI like pacemaker and metallic implants are also an important issue as detailed history of all these issues will not be possible in acute stroke patients. ${ }^{13,14}$

According to present recommendations, NCCT brain is the imaging modality of choice in acute stroke patients owing to easy availability, quick and high sensitivity to rule out hemorrhage.

\section{Routine Use of MRI Brain to Exclude Cerebral Microbleeds before Administration of Intravenous Thrombolysis Is Not Recommended (I-B)}

Cerebral microbleeds are basically hemosiderin deposits in perivascular spaces secondary to the leakage from the small vessels of the brain. This is usually detected on susceptibility-weighted imaging-magnetic resonance imaging sequences as homogenously hypointense areas measuring less than $10 \mathrm{~mm}$ in largest dimension. They can be observed in normal patients and sometimes pathological states like cerebral amyloid angiopathy, or Alzheimer disease. Presence of the microbleeds on imaging has shown to be one of the risk factors for ICH. Certainly, this is one of the important considerations in stroke patients receiving thrombolysis/antithrombotic therapy. ${ }^{15}$ Multiple factors play important role in evaluation of cerebral microbleeds in relation to risk of ICH after thrombolysis/antithrombotic therapy. Baseline clinical risk factors, number of microbleeds, and location of these lesions can guide the clinician in the riskbenefit analysis. ${ }^{16}$

The reported risk of symptomatic intracerebral hemorrhage $(\mathrm{sICH})$ in patients with microbleeds after IVT is to be 5 to $6.5 \%$, according to ECASS and NINDS trials. However, there is no RCT of IVT risk versus benefit in patients of cerebral microbleeds on MRI. So, withholding the treatment based on cerebral microbleeds is not recommended according to recent American Heart Association/American Stroke Association (AHA/ASA) guidelines. Even though presence of cerebral microbleeds is a risk for sICH after IVT, benefit outweighs the risk in these patients. Hence, screening of acute 
stroke patients for the baseline cerebral microbleeds is not recommended before initiating thrombolysis treatment. $6,17,18$

\section{Patients Who Are Potential Candidate for Mechanical Thrombectomy, Noninvasive Vascular Imaging (CTA/MRA) Is Recommended during Initial Imaging Evaluation (I-A)}

Ideal imaging technique is one which is quick, reliable, widely available, as clinical outcome after ET is dependent on time as well as amount of salvageable brain tissue. Every 30 minutes of delay in the treatment might increase risk of poor outcome by $\sim 15 \%{ }^{4}$

Clinical benefit of endovascular MT in acute stroke patients has been demonstrated in 6 RCTs whenever the procedure is being performed in less than 6 hours from onset of symptoms. The procedure is targeted to the large vessel occlusion (LVO) subgroup of acute stroke patients. ${ }^{19-24}$ Even though clinical parameters like National Institutes of Health Stroke Scale (NIHSS) help the identify the stroke patient with LVO, all 6 RCTs have used either computed tomography angiography/magnetic resonance imaging (CTA/MRA) as imaging modality for the same purpose. Of these, three trials actually incorporated some form of additional imaging (collateral flow and perfusion imaging) in their protocol. $^{20,21,24}$ The Mechanical Thrombectomy After Intravenous Alteplase Versus Alteplase Alone After Stroke (THRACE) and Multicenter Randomized Clinical Trial of Endovascular Treatment of Acute Ischemic Stroke in the Netherlands (MR CLEAN) trials just had CTA/MRA in their protocol in addition to CT/MRI imaging to rule out hemorrhage. ${ }^{19,22}$ These two trials independently showed clinical benefit in the treated arm same as other 4 RCTs using additional imaging.

Multiphase CT angiography is new acute stroke imaging technique, which was used in the Endovascular Treatment for Small Core and Anterior Circulation Proximal Occlusion with Emphasis on Minimizing CT to Recanalization Times (ESCAPE) trial for the patient selection in ET arm. This technique basically involves acquiring two additional scans after routine CT angiography, with delay of 8 seconds in between the scans (4 seconds for table positioning and 4 seconds for scan acquisition) in early venous phase and late venous phase. These additional scans were targeted only from vortex to skull base region to assess the pial collateral filling. Patients were selected if there is no phase delay or one phase delay in the filling of peripheral leptomeningeal vessels in the affected cerebral hemisphere in comparison to the contralateral normal brain parenchyma. This technique has several advantages in comparison to CT perfusion (CTP) as no need for extra IV contrast, quick without any additional postprocessing needed to generate data and less radiation. Multiphase CT angiography and CTP have similar impact on clinical decision making on patient selection as well as predicting clinical outcome after ET., 49,20,25 Whenever feasible, multiphase CT angiography could be important tool in acute stroke imaging, which provides all the necessary information required in decision making of ET (II-C). ${ }^{18}$

In AIS patients of onset 0 to 6 hours, the role of additional imaging in the form of perfusion imaging is not well established and not recommended. It could actually lead to exclusion of patients who otherwise would benefit from the endovascular treatment. ${ }^{25}$

For acute stroke patients of 6 to 24 hours symptoms onset, diffusion-weighted imaging (DWI) or CTP Assessment with Clinical Mismatch in the Triage of Wake-Up and Late Presenting Strokes Undergoing Neurointervention with Trevo (DAWN) and The Endovascular Therapy Following Imaging Evaluation for Ischemic Stroke (DEFUSE 3) demonstrated significant clinical benefit of endovascular treatment. The DWAN trial used clinical-core mismatch (combination of age-adjusted NIHSS score and age-adjusted core infarct volume measured on CTP/DWI-MRI) as eligibility criteria for patient selection in endovascular treatment arm. This trial demonstrated clinical benefit in terms of functional outcome at 90 days in endovascular treatment arm ( 49 vs. $13 \%$ in control arm). ${ }^{26}$

DEFUSE 3 trial used perfusion-core mismatch and core volume based on imaging as eligibility criteria to select patients of acute stroke in endovascular arm. DEFUSE 3 also showed benefit in functional outcome at 90 days in the endovascular arm (44.6 vs. $16.7 \%$ in control arm). ${ }^{27}$ Both these RCTs had included additional imaging in the form of perfusion imaging (CTP/MRP) for the patient selection in patients of 6 to 24 hours stroke onset who are potential candidate for endovascular MT.28

In conclusion, stroke imaging protocol for any stroke onset 0 to 6 hours should include CT/MRI to exclude hemorrhage and CTA/MRA of both intracranial and extracranial vessels to exclude LVO. In addition to this, CTA/MRA helps

Table 1 Acute stroke randomized control trials showing time window and Imaging modalities used

\begin{tabular}{|l|l|l|l|}
\hline \multicolumn{2}{|c|}{ Name of the trial } & $\begin{array}{l}\text { Time window from stroke onset } \\
\text { (hours) }\end{array}$ & Imaging modality \\
\hline 1 & MR CLEAN (2015) & $0-6$ & CT, CTA or MR, MRA \\
\hline 2 & ESCAPE (2015) & $0-12$ & CT, mCTA (multiphase CTA) \\
\hline 3 & REVASCAT (2015) & $0-8$ & CT, CTA OR MR, MRA \\
\hline 4 & SWIFT PRIME (2015) & $0-6$ & CT, CTA, CTP Or MRA, MRP \\
\hline 5 & EXTEND IA (2015) & $0-6$ & CT, CTA, CTP \\
\hline 6 & THRACE (2016) & $0-5$ & CT, CTA OR MRI, MRA \\
\hline 7 & DAWN (2018) & $6-24$ & CT, CTA, CTP Or MRI, MRA, MRP \\
\hline 8 & DEFUSE-3 (2018) & $6-16$ & CT, CTA, CTP \\
\hline
\end{tabular}


neurointerventionist to plan the treatment more effectively and rapidly. Multiphase CTA could be important tool in addition to single-phase CTA predicting patient selection and clinical outcome after ET. Stroke onset of 6 to 24 hours, imaging protocol should include perfusion imaging (CTP/ MRP) or DWI infarct core-clinical mismatch to identify potential candidates for endovascular MT ( - Table 1 ).

\section{Routine Screening of Serum Creatinine Is Not Recommended for CT Angiography in Patients without Prior Renal Impairment or on Dialysis (Ila-B)}

Use of CTA imaging modality in acute stroke patients needs administration of contrast media. As benefit of stroke intervention is time dependent, waiting for the serum creatinine value in window period before proceeding for CTA is not advocated. On the other hand, contrast-induced renal injury and potential interference of contrast media on IV thrombolysis effect are major issues. ${ }^{17}$ Multiple retrospective studies have reported safety of CT contrast media in patients with normal as well as abnormal serum creatinine values. Aulický et al in their observation cohort study reported no significant serum creatinine increase or increased rates of sICH (in IV thrombolysis cases) in the patients received CT contrast compared with control population. ${ }^{29}$ All these studies used direct administration of IV contrast without any additional renoprotective methods. Still, no significant effects in terms of acute renal injury or need for dialysis were reported. ${ }^{29,30}$

IV contrast media can be safely used in patients with AIS patients for angiographic/perfusion study especially in the patient without any history of renal impairment/on dialysis. In the patients with renal impairment/on dialysis, alternate imaging modality like MRA (noncontrast) can be considered.

\section{Wake-Up Stroke/Stroke of Unknown Onset Patients; MRI Brain for the DWI-FLAIR Mismatch Is Reasonable to Predict Salvageable Brain Tissue, Which Benefit in Decision Making during Emergency Stroke Management (Ila-B)}

Wake-up trial investigated the role of diffusion-weighted imaging-fluid attenuated inversion recovery (DWI-FLAIR) mismatch based IVT in the patient with wake-up stroke/stroke of unknown onset. They selected patients based on DWI hyperintensity with corresponding absent changes on FLAIR imaging in these subtype of stroke patients. The trial showed favorable outcome in the thrombolysis arm compared with the control arm. They concluded that DWI-FLAIR mismatch may suggest stroke onset of less than 4.5 hours and potential candidate for IV thrombolysis. ${ }^{31}$

Few other studies have reported $245 \%$ of FLAIR positive patients had stroke onset of less than 4.5 hours. But, DWI-FLAIR mismatch had high specificity and positive predictive value to predict stroke onset at both $1.5 \mathrm{~T}$ and 3 T MRI. ${ }^{32,33}$ Even though FLAIR imaging has overall less sensitivity in prediction of stroke onset, DWI-FLAIR mismatch has high specificity and should be considered as selection criteria before IVT in all stroke patients with unknown time onset.

\section{Part II: Mechanical Thrombectomy Guidelines}

\section{Mechanical Thrombectomy in Anterior Circulation with Large Vessel Occlusion (LVO) Less Than 6 Hours and 6 to 24 Hours (I-A)}

In patients with anterior circulation major or large vessel occlusion-related AIS presenting within 6 hours after symptom onset, we recommend MT with or without IVT over best medical management alone to improve functional outcome. MT with stent retrievers is recommended over intraarterial fibrinolysis as first-line therapy. ${ }^{19-24}$

Direct aspiration thrombectomy as first-pass MT is recommended as noninferior to stent retriever for patients who meet all the criteria that are applicable for stent retriever thrombectomy. ${ }^{34}$

Patients should receive MT if they meet all the following criteria

(1) Prestroke mRS score of 0 to 1 ; (2) causative occlusion of the internal carotid artery or middle cerebral artery (MCA) segment 1 (M1); (3) age $\geq 18$ years; (4) NIHSS score of $\geq 6$; (5) ASPECTS of $\geq 6$; and (6) treatment can be initiated (groin puncture) within 6 hours of symptom onset.

Administration of intravenous tissue-type plasminogen activator (IV-tPA) does not exclude eligible patients from receiving ET. In fact, recent evidence suggests that patients treated with ET plus IV-tPA might have better functional outcomes, lower mortality, and higher rate of successful recanalization without having increased odds of $\mathrm{sICH} .{ }^{25}$

Further, there should be no delay to assess the effect of IV-tPA following administration (III-B). In agreement with AHA guidelines, patients who meet the criteria for on label use of IV-tPA should receive IV-tPA, irrespective of whether endovascular treatments are being offered. ${ }^{17,35}$ Current AHA/ASA guidelines do not recommend the use of IA thrombolysis over MT with stent retrievers/contact aspiration as first-line therapy for the treatment of AIS due to LVO. However, the use of intra-arterial thrombolysis as a salvage technique is acceptable to achieve modified thrombolysis in cerebral infarction grade (mTICI) $\geq 2 \mathrm{~b}$ revascularization. ${ }^{36}$ Currently, there is no standardized protocol for dose of intraarterial thrombolytics; however, the lowest dose necessary to achieve recanalization should be used and the drug infusion must be administered slowly and carefully (usually over 10-20 minutes).

\section{To Ensure Benefit, Reperfusion to mTICl Grade} 2b/3 Should Be Achieved as Early as Possible within the Therapeutic Window (I-A)

Recanalization is the single most important prognostic factor for good functional outcome in patients with AIS. All recent endovascular trials used the mTICI $2 b / 3$ threshold for adequate reperfusion, with high rates achieved.

Studies have shown that patients with mTICI grade $2 \mathrm{~b} / 3$ recanalization, $41.0 \%$ were discharged home compared with $17.4 \%$ of the mTICI grade $2 \mathrm{a}$ group. With respect to functional outcome at 90 days, $34 \%$ of patients with TICI $2 \mathrm{a}$ 
had a mRS score of 0 to 2 at 90 days versus $49 \%$ with a TICI grade of $2 \mathrm{~b} / 3 . .^{37,38}$

In Highly Effective Reperfusion evaluated in Multiple Endovascular Stroke Trials (HERMES), 402 of 570 patients (71\%) were successfully reperfused to TICI $2 \mathrm{~b} / 3$ that correlated to overall good outcome. ${ }^{25}$

In the 6 to 24-Hour Time Window, We Recommend Mechanical Thrombectomy with Large Vessel Occlusion-Related Anterior Circulation Stroke Fulfilling the Selection Criteria of DEFUSE-3* or DAWN**, Including Estimated Volume of Infarct Core (I-A)

*6 to 16 hours since time last known well:

- Age $\leq 90$ years and NIHSS $\geq 6$ : infarct core volume $15 \mathrm{~mL}$ and penumbra volume/core vol $>1.8$.

${ }^{* *} 6$ to 24 hours since time last known well:

- Age <80 years: infarct core volume $30 \mathrm{~mL}$ if NIHSS $\geq 10$; infarct core $\leq 51 \mathrm{~mL}$ if NIHSS score $\geq 20$.

- Age $\geq 80$ years: infarct core $\leq 20 \mathrm{~mL}$ and NIHSS score $\geq 10$.

The DAWN and DEFUSE trials suggested a change in the treatment paradox of "time is brain" to "collaterals are brain." Time is still a determinant of outcome, but some patients with collaterals are able to stand the test of time longer than others.

DAWN and DEFUSE 3 are the only RCTs showing benefit of MT $>6$ hours from onset. Therefore, only the eligibility criteria from one or the other of these trials should be used for patient selection. ${ }^{26,27}$ Both trials clearly demonstrated an overall benefit in functional outcome at 90 days in the treatment group.

In Patients of Acute Ischemic Stroke (AIS) Who Undergo Endovascular Thrombectomy, It Is

Recommended to Maintain the BP at $\leq 180 / 105 \mathrm{~mm} \mathrm{Hg}$ during and for 24 Hours Post Procedure (Ila-B)

Labetalol or an IV $\beta$-blocker such as metoprolol in low doses is recommended as the preferred drug of choice to reduce blood pressure to recommended levels before MT. ${ }^{20}$

The DAWN protocol recommends maintaining systolic blood pressure $<140 \mathrm{~mm} \mathrm{Hg}$ in the first 24 hours in subjects who are reperfused after MT (defined as achieving more than two-thirds MCA territory reperfusion). Further studies are needed to determine the optimal BP target during and after MT. ${ }^{26}$

Anesthetic Choice during EVT for AIS on the Basis of Individualized Assessment of Patient Risk Factors, Technical Performance of the Procedure, and Other Clinical Characteristics (Ila-B)

Anesthetic choice for patients receiving MT for AIS has historically been a topic of great controversy and confusion. Conscious sedation (CS) was the anesthetic modality widely used during endovascular procedures for acute stroke in the recent endovascular trials.

Three smalls ( $\leq 150$ participants each) single-center RCTs have compared general anesthesia (GA) with CS during acute stroke endovascular procedures. All failed to show superiority of GA for the primary end point ( 2 clinical, 1 DW-MRI infarct growth), whereas two of the three showed better outcomes for GA for some of the many secondary clinical end points..$^{39-41}$

There is conflicting evidence regarding the ideal anesthesia management of patients with AIS, with several retrospective studies showing benefit of non-GA over GA, and three RCTs (SIESTA, ANSTROKE, GOLIATH) showing marginal benefit of GA over non-GA.

Until further data are available, either method of procedural sedation for acute stroke endovascular procedures is reasonable.

The Use of a Proximal Balloon Guide Catheter (BGC) or a Large-Bore Distal-Access Catheter, Rather Than a Cervical Guide Catheter Alone, in Conjunction with Stent Retrievers May Be Beneficial (Ila-C)

Balloon guide catheters (BGCs) are used as an aid in achieving proximal flow arrest and thus avoid distal embolization to new territory. In vitro experience shows that proximal flow arrest during clot retrieval results in less clot fragmentation and less unwanted distal embolization. ${ }^{42} \mathrm{~A}$ recent meta-analysis by Brinjikji et al with 2022 patients (1083 BGC group and 939 non BGC group) reported better technical and clinical outcomes with BGC. Mean procedure time was also significantly shorter for BGC-treated patients (weighted mean difference of $\sim 7.7$ minutes $).{ }^{43}$

Mechanical Thrombectomy May Be Reasonable Option for Carefully Selected Patients with AIS in Whom Treatment Can Be Initiated within 6 Hours of Symptom Onset Secondary to Occlusion of the MCA Segment 2 (M2) or MCA Segment 3 (M3) Portion of the MCAs (IIb-B)

In HERMES meta-analysis, which included the five trials MR CLEAN, ESCAPE, REVASCAT, SWIFT PRIME, and EXTEND-IA, the direction of treatment effect for MT over standard care was favorable in M2 occlusions, but the adjusted crude odd ratio was not significant (1.28 [95\% confidence interval, CI: $0.51-3.21]){ }^{25}$

Distal arterial occlusions or branch occlusion usually compromise small brain territories but ischemic cores could include highly eloquent areas (e.g., motor cortex, supplementary motor area, Broca's area) that potentially leave severe disabling clinical deficits to the patient. Current evidence suggests that perfusion study plays important role in decision making over the Alberta Stroke Program Early CT Score (ASPECTS) and GA is preferred over CS for distal artery recanalization.

Mechanical Thrombectomy with Stent Retrievers May Be Reasonable for Patients with AIS within 6 Hours of Symptom Onset and Who Have Prestroke mRS Score $>1$, ASPECTS $<6$, or NIHSS Score $<6$ and Causative Occlusion of the Internal Carotid artery (ICA) or Proximal MCA (M1) (IIb-B)

In general, patients with prestroke $m R S$ of $\geq 2$ do poorly with MT. This also includes patients with cancer, advanced age, and previous major strokes. ${ }^{44}$ It is often important to 
determine the baseline functional status before initiating stroke transfers, especially in patients with premorbid conditions or those who are already hospitalized. The other significant proportion of patients with poor functional status is the elderly or those $\geq 80$ years of age. Most trials that have shown poor outcome in these subgroups are because of their preexisting poor mRS. Nevertheless, it is still beneficial to consider MT in a selected group of octogenarians as well as nonagenarians who have good baseline functional status.

The NIHSS has been shown to be a predictor of both short- and long-term outcome of stroke patients. The natural history of LVO strokes associated with low NIHSS may not be benign, as nearly a quarter of patients without recanalization did not achieve independence at 90 days. In a large prospective study that was part of the Screening Technology and Outcomes Project in Stroke, it was found that NIHSS score of greater than 10 had an $81 \%$ positive predictive value for LVO and also nearly $90 \%$ of patients without proximal arterial occlusions presented with NIHSS scores $\leq 10$, so did $55 \%$ of all patients found to have occlusive lesions that may have been amenable for interventional therapy ${ }^{45}$

\section{Treatment of Tandem Occlusions (Both Extracranial and Intracranial Occlusions) When Performing Mechanical Thrombectomy May Be Reasonable (IIb-B)}

Multiple retrospective case series have shown the technical success of MT for tandem occlusions but do not provide specifics on comparative approaches. Tandem occlusions were included in many recent endovascular trials that showed benefit of MT over medical management alone..$^{20,22}$

It is unclear what is the best treatment sequence to recanalize tandem occlusion, proximal first versus distal first. Proximal occlusions (carotid and vertebral) can sometimes be treated with good angioplasty alone. When successful, this approach lessens the need for dual antiplatelet therapy and the risk of hemorrhagic complications.

From whatever data available, acute stent placement during MT of patients with tandem occlusion appears to have lower-than-expected rates of adverse events. One of the main challenges for patients with tandem occlusions is the need for antiplatelet agents after placing the stent in addition to the IVT that are often times already infused. Pooled analysis showed favorable outcome in $~ 44 \%$ of patients, with $7 \%$ sICH rate, but the higher rate of ICH did not impact mortality. ${ }^{46}$

The choice of antiplatelet agent may vary from aspirin monotherapy for 24 hours or initiate IIb/IIla receptor antagonist for 12 hours followed by loading of dual antiplatelets after check CT scan. Most centers use tirofiban or eptifibatide over abciximab according to the recently published 2019 stroke guidelines suggest that tirofiban and eptifibatide may have better safety profile in patients with AIS due to their shorter half-life. ${ }^{18}$
Preliminary studies indicate that tirofiban is an effective bridge to oral dual antiplatelet therapy and is not associated with increased risk of intracranial hemorrhage in acute stroke. ${ }^{47-49}$

\section{Mechanical Thrombectomy May Be Reasonable for Carefully Selected Patients with AIS in Whom Treatment Can Be Initiated within 6 Hours of Symptom Onset and Who Have Causative Occlusion of the Anterior Cerebral Arteries, Vertebral Arteries, Basilar Artery, or Posterior Cerebral Arteries (IIb-C)}

Recanalization is the single most important prognostic factor for good functional outcome in patients with AIS in the posterior circulation. For basilar artery stroke, there are no published randomized clinical trial results. An international prospective registry of patients with basilar artery occlusion did not show the superiority of endovascular thrombectomy (EVT) over IVT. ${ }^{50}$ Few other multicenter registries have reported safety and equally beneficial role of EVT in patients of posterior circulation stroke secondary to occlusion of basilar artery, distal vertebral artery, and posterior cerebral artery in comparison to anterior circulation LVO. ${ }^{51-53}$ The predominant determinants of good clinical outcome are low NIHSS at presentation, good collaterals, pcASPECT $\geq 8$, younger age, and faster onset to recanalization time. Presence of significant brainstem infarction and comatose status of the patient $>4$ hours are invariably associated with poor outcome. The ENDOSTROKE registry even reported good clinical outcome in 35\% of the patients when EVT was done beyond 6 hours and $24 \%$ of patients with unknown time of onset. They concluded that salvageable tissue on MRI and collateral status determine outcome than just time of onset and deferring EVT only based on time is not justified in posterior circulation stroke..$^{53}$ There is a consensus among the experts that in analogy to anterior circulation major artery occlusion and with regard to the poor outcome of acute basilar artery occlusions, the therapeutic approach with IVT plus MT should be strongly considered.

Limited evidence suggests that direct contact aspiration MT is faster and safer than stentrievers techniques for posterior circulation strokes. However, the best approach/device/technique should always be decided on case-to-case basis, considering additional factors such as the presence of atherosclerosis in the vertebrobasilar system, collateral circulation, and anatomical variations.

The promising results with second- and third-generation devices have expanded their use to more distal smaller vessels like anterior and posterior cerebral arteries. ${ }^{54}$

\section{For Adults with Large Vessel Occlusion-Related Acute Ischemic Stroke, Intravenous Thrombolysis Plus Mechanical Thrombectomy Compared with Mechanical Thrombectomy Alone Improve Functional Outcome (I-A)}

A systematic review and meta-analysis of 13 studies allowing thenonrandomized comparison of MT plusIVTversus MTalone 
in adults with anterior circulation LVO-related AIS suggested a superiority of MT + IVT for functional independence (mRS score $\leq 2$ : odds ratio [OR]: $\left.1.27,95 \% \mathrm{CI}: 1.05-1.55 ; \mathrm{I}^{2}=17 \%\right) .55$ Another meta-analysis did not suggest the superiority of MT + IVT versus MT alone in the subgroup of patients eligible for IVT (mRS score $\leq 2$ : OR: $0.93,95 \% \mathrm{CI}$ : 0.57-1.49; $\mathrm{I}^{2}$ $=41 \%) \cdot{ }^{56}$ Multicenter RCTs comparing these two treatment approaches will provide definitive information on the efficacy and safety of direct MT compared with the combined approach of IV-tPA followed by MT.

\section{Role of IV Glycoprotein IIb/IIla Inhibitors Administered during Endovascular Stroke Treatment Are Uncertain (IIb-C)}

The published literature is limited primarily to case series and retrospective reviews and focuses largely on administration of IV glycoprotein IIb/IIIa inhibitors to prevent thrombus formation during emergent extracranial or intracranial artery stenting and MT. ${ }^{57-59}$ Further multicenter analyses of endovascular stroke therapy are needed necessitating adjunctive antiplatelet therapy for emergent stenting.

\section{Training Requirement and Minimum Qualification of Stroke Physicians for Performing Endovascular Therapy in Acute Ischemic Stroke Patients}

Diagnosis and emergency management of AIS are often challenging and they require inputs from the expertise of multiple specialties. Integrated approach involving history, clinical examination, and neuroimaging guides the stroke physician to decide proper treatment plan foe particular patient. In addition, patient selection and procedural expertise are of paramount importance to achieve good clinical outcome for EVT in ischemic stroke. In multiple RCTs favoring the benefit of EVT in ischemic stroke, procedures were performed by experienced neurointerventionist (interventional neuroradiology, endovascular neurosurgeon, interventional neurologist). ${ }^{19-27}$ Hence, training guidelines play important role in maintaining quality standards for physicians performing EVT in ischemic stroke.

International multisociety consensus on training guidelines for endovascular intervention in AIS has proposed that physician should have baseline residency training (radiology, neurosurgery, and neurology) in stroke imaging, neuroangiography, and its interpretation under supervision of neuroradiologist. After that, advanced training in neurointervention for dedicated time period of minimum 1 year is recommended that should include:

a. Trainee should have participated in at least 150 to 250 therapeutic neuroendovascular procedures, of which he/she should be primary operator in half of the cases.

b. Trainee should have participated in at least 30 to 50 MT procedures, of which he/she should be primary operator in at least half.

Physician performing EVT in ischemic stroke should also participate in continue medical education on AIS. The quality assurance in terms of remote and long-term outcome of these procedures should be done as per international guidelines. ${ }^{60-62}$ In India, presently we do not have any multisociety consensus guidelines regarding the training as well as quality assurance for physicians performing EVT in AIS.

\section{Conclusion}

Appropriate patient selection and expedient recanalization are the mainstay of modern management of AIS. Only a minority of patients (7-15\%) of patients are eligible for ET. Patient selection may be time based or perfusion based. Central to both paradigms is the selection of a patient with a small core, a significant penumbra that can be differentiated from areas of oligemia based on the appropriate imaging. Till recently, the mainstay of management of AIS has been IVT. However, response to treatment and outcomes in the presence of a large vessel occlusion were largely suboptimal. Endovascular thrombectomy techniques with stentrievers and aspiration catheters have revolutionized stroke treatment significantly improving outcomes in this once untreatable disease. The development of in-hospital acute stroke treatment pathway and evidence-based protocols are important aspects of appropriate imaging as well as interventions in these patients.

\section{Financial Support and Sponsorship}

Nil.

\section{Conflicts of Interest}

There are no conflicts of interest.

\section{References}

1 Lees KR, Emberson J, Blackwell L, et al. Stroke Thrombolysis Trialists' Collaborators Group. Effects of alteplase for acute stroke on the distribution of functional outcomes. Stroke 2016;47(9):2373-2379

2 Aghaebrahim A, Streib C, Rangaraju S, et al. Streamlining door to recanalization processes in endovascular stroke therapy. J Neurointerv Surg 2017;9(4):340-345

3 Deguchi I, Mizuno S, Kohyama S, Tanahashi N, Takao M. Dripand-Ship thrombolytic therapy for acute ischemic stroke. J Stroke Cerebrovasc Dis 2018;27(1):61-67

4 Menon BK, Sajobi TT, Zhang Y, et al. Analysis of workflow and time to treatment on thrombectomy outcome in the endovascular treatment for small core and proximal occlusion ischemic stroke (ESCAPE) randomized, controlled trial. Circulation 2016;133(23):2279-2286

5 National Institute of Neurological Disorders and Stroke rt-PA Stroke Study Group. Tissue plasminogen activator for acute ischemic stroke. N Engl J Med 1995;333(24):1581-1587

6 Hacke W, Kaste M, Bluhmki E, et al. ECASS Investigators. Thrombolysis with alteplase 3 to 4.5 hours after acute ischemic stroke. N Engl J Med 2008;359(13):1317-1329

7 Hand PJ, Wardlaw JM, Rowat AM, Haisma JA, Lindley RI, Dennis MS. Magnetic resonance brain imaging in patients with acute stroke: feasibility and patient related difficulties. J Neurol Neurosurg Psychiatry 2005;76(11):1525-1527 
8 Christensen AF, Christensen H. Editorial: imaging in acute stroke-new options and state of the art. Front Neurol 2018;8:736

9 Rai N, Prasad K, Bhatia R, et al. Development and implementation of acute stroke care pathway in a tertiary care hospital in India: a cluster-randomized study. Neurol India 2016;64(Suppl):S39-S45

10 Martínez-Sánchez P, Fuentes B, Medina-Báez J, et al. [Development of an acute stroke care pathway in a hospital with stroke unit]. Neurologia 2010;25(1):17-26

11 Wang H, Thevathasan A, Dowling R, Bush S, Mitchell P, Yan B. Streamlining workflow for endovascular mechanical thrombectomy: lessons learned from a comprehensive stroke center. J Stroke Cerebrovasc Dis 2017;26(8):1655-1662

12 Macellari F, Paciaroni M, Agnelli G, Caso V. Neuroimaging in intracerebral hemorrhage. Stroke 2014;45(3):903-908

13 Kidwell CS, Chalela JA, Saver JL, et al. Comparison of MRI and CT for detection of acute intracerebral hemorrhage. JAMA 2004;292(15):1823-1830

14 Chalela JA, Kidwell CS, Nentwich LM, et al. Magnetic resonance imaging and computed tomography in emergency assessment of patients with suspected acute stroke: a prospective comparison. Lancet 2007;369(9558):293-298

15 Wang Z, Soo YO, Mok VC. Cerebral microbleeds: is antithrombotic therapy safe to administer? Stroke 2014;45(9):2811-2817

16 Haller S, Vernooij MW, Kuijer JPA, Larsson E-M, Jäger HR, Barkhof F. Cerebral microbleeds: imaging and clinical significance. Radiology 2018;287(1):11-28

17 Powers WJ, Derdeyn CP, Biller J, etal.American Heart Association Stroke Council. 2015 American Heart Association/American Stroke Association Focused Update of the 2013 Guidelines for the early management of patients with acute ischemic stroke regarding endovascular treatment: a guideline for healthcare professionals from the American Heart Association/ American Stroke Association. Stroke 2015;46(10):3020-3035

18 Powers WJ, Rabinstein AA, Ackerson T, et al. American Heart Association Stroke Council. 2018 Guidelines for the early management of patients with acute ischemic stroke: a guideline for healthcare professionals from the American Heart Association/ American Stroke Association. Stroke 2018;49(3):e46-e110

19 Berkhemer OA, Fransen PSS, Beumer D, et al. MR CLEAN Investigators. A randomized trial of intraarterial treatment for acute ischemic stroke. N Engl J Med 2015;372(1):11-20

20 Goyal M, Demchuk AM, Menon BK, et al. ESCAPE Trial Investigators. Randomized assessment of rapid endovascular treatment of ischemic stroke. $\mathrm{N}$ Engl J Med 2015;372(11):1019-1030

21 Saver JL, Goyal M, Bonafe A, et al. SWIFT PRIME Investigators. Stent-retriever thrombectomy after intravenous t-PA vs. t-PA alone in stroke. N Engl J Med 2015;372(24):2285-2295

22 Bracard S, Ducrocq X, Mas JL, et al. THRACE investigators. Mechanical thrombectomy after intravenous alteplase versus alteplase alone after stroke (THRACE): a randomised controlled trial. Lancet Neurol 2016;15(11):1138-1147

23 Jovin TG, Chamorro A, Cobo E, etal. REVASCATTrial Investigators. Thrombectomy within 8 hours after symptom onset in ischemic stroke. N Engl J Med 2015;372(24):2296-2306

24 Campbell BC, Mitchell PJ, Kleinig TJ, et al. EXTEND-IA Investigators. Endovascular therapy for ischemic stroke with perfusion-imaging selection. $\mathrm{N}$ Engl J Med 2015;372(11):1009-1018

25 Goyal M, Menon BK, van Zwam WH, et al. HERMES collaborators. Endovascular thrombectomy after large-vessel ischaemic stroke: a meta-analysis of individual patient data from five randomised trials. Lancet 2016;387(10029):1723-1731

26 Nogueira RG, Jadhav AP, Haussen DC, et al. DAWN Trial Investigators. Thrombectomy 6 to 24 hours after stroke with a mismatch between deficit and infarct. $N$ Engl J Med 2018;378(1):11-21

27 Albers GW, Marks MP, Kemp S, et al. DEFUSE 3 Investigators. DEFUSE 3 Investigators. Thrombectomy for stroke at 6 to 16 hours with selection by perfusion imaging. N Engl J Med 2018;378(8):708-718

28 GG SK, Nagesh CP. Acute ischemic stroke: a review of imaging, patient selection, and management in the endovascular era. Part I: initial management and imaging. J Clin Interv Radiol ISVIR 2018;2:155-168

29 Aulický P, Mikulík R, Goldemund D, Reif M, Dufek M, Kubelka T. Safety of performing CT angiography in stroke patients treated with intravenous thrombolysis. J Neurol Neurosurg Psychiatry 2010;81(7):783-787

30 Ehrlich ME, Turner HL, Currie LJ, Wintermark M, Worrall BB, Southerland AM. Safety of computed tomographic angiography in the evaluation of patients with acute stroke. Stroke 2016;47(8):2045-2050

31 Thomalla G, Simonsen CZ, Boutitie F, et al. WAKE-UP Investigators. MRI-guided thrombolysis for stroke with unknown time of onset. N Engl J Med 2018;379(7):611-622

32 Ebinger M, Galinovic I, Rozanski M, Brunecker P, Endres M, Fiebach JB. Fluid-attenuated inversion recovery evolution within 12 hours from stroke onset: a reliable tissue clock? Stroke 2010;41(2):250-255

33 Emeriau S, Serre I, Toubas O, Pombourcq F, Oppenheim C, Pierot L. Can diffusion-weighted imaging-fluid-attenuated inversion recovery mismatch (positive diffusion-weighted imaging/negative fluid-attenuated inversion recovery) at 3 Tesla identify patients with stroke at $<4.5$ hours? Stroke 2013;44(6):1647-1651

34 Lapergue B, Blanc R, Gory B, et al. ASTER Trial Investigators. Effect of endovascular contact aspiration vs stent retriever on revascularization in patients with acute ischemic stroke and large vessel occlusion: The ASTER randomized clinical trial. JAMA 2017;318(5):443-452

35 Jauch EC, Saver JL, Adams HP Jr, et al. American Heart Association Stroke CouncilCouncil on Cardiovascular NursingCouncil on Peripheral Vascular DiseaseCouncil on Clinical Cardiology. Guidelines for the early management of patients with acute ischemic stroke: a guideline for healthcare professionals from the American Heart Association/American Stroke Association. Stroke 2013;44(3):870-947

36 Dippel DW, Majoie CB, Roos YB, et al. MR CLEAN Investigators. for the MR CLEAN Investigators. Influence of device choice on the effect of intra-arterial treatment for acute ischemic stroke in MR CLEAN (Multicenter Randomized Clinical Trial of Endovascular Treatment for Acute Ischemic Stroke in the Netherlands. Stroke 2016;47(10):2574-2581

37 Higashida RT, Furlan AJ, Roberts $\mathrm{H}$, et al. Technology Assessment Committee of the American Society of Interventional and Therapeutic NeuroradiologyTechnology Assessment Committee of the Society of Interventional Radiology. Trial design and reporting standards for intra-arterial cerebral thrombolysis for acute ischemic stroke. Stroke 2003;34(8):e109-e137

38 Broderick JP, Palesch YY, Demchuk AM, et al. Interventional Management of Stroke (IMS) III Investigators. Endovascular therapy after intravenous t-PA versus t-PA alone for stroke. N Engl J Med 2013;368(10):893-903

39 Löwhagen Hendén P, Rentzos A, Karlsson JE, et al. Hypotension during endovascular treatment of ischemic stroke is a risk factor for poor neurological outcome. Stroke 2015;46(9):2678-2680

40 Schönenberger S, Uhlmann L, Hacke W, et al. Effect of conscious sedation vs general anaesthesia on early neurological improvement among patients with ischemic stroke undergoing endovascular thrombectomy: a randomized clinical trial. JAMA 2016;316(19):1986-1996 
41 Simonsen CZ, Yoo AJ, Sørensen LH, et al. Effect of general anesthesia and conscious sedation during endovascular therapy on infarct growth and clinical outcomes in acute ischemic stroke: a randomized clinical trial. JAMA Neurol 2018;75(4):470-477

42 Chueh JY, Kühn AL, Puri AS, Wilson SD, Wakhloo AK, Gounis MJ. Reduction in distal emboli with proximal flow control during mechanical thrombectomy: a quantitative in vitro study. Stroke 2013;44(5):1396-1401

43 Brinjikji W, Starke RM, Murad MH, et al. Impact of balloon guide catheter on technical and clinical outcomes: a systematic review and meta-analysis. J Neurointerv Surg 2018;10(4):335-339

44 Lindvig K, Møller H, Mosbech J, Jensen OM. The pattern of cancer in a large cohort of stroke patients. Int J Epidemiol 1990;19(3):498-504

45 Maas MB, Furie KL, Lev MH, et al. National Institutes of Health Stroke Scale score is poorly predictive of proximal occlusion in acute cerebral ischemia. Stroke 2009;40(9):2988-2993

46 Sivan-Hoffmann R, Gory B, Armoiry X, et al. Stent-retriever thrombectomy for acute anterior ischemic stroke with tandem occlusion: a systematic review and meta-analysis. Eur Radiol 2017;27(1):247-254

47 Lee JI, Gliem M, Gerdes G, et al. Safety of bridging antiplatelet therapy with the gpIIb-IIIa inhibitor tirofiban after emergency stenting in stroke. PLoS One 2017;12(12):e0190218

48 Zhu YQ, Zhang YJ, Ruan HL, Liu Q, Zhan Q, Li Q. Safety of tirofiban for patients with acute ischemic stroke in routine clinical practice. Exp Ther Med 2015;10(1):169-174

49 Siebler M, Hennerici MG, Schneider D, et al. Safety of tirofiban in acute ischemic stroke: the SaTIS trial. Stroke 2011;42(9):2388-2392

50 Schonewille WJ, Wijman CA, Michel P, et al. BASICS study group. Treatment and outcomes of acute basilar artery occlusion in the Basilar Artery International Cooperation Study (BASICS): a prospective registry study. Lancet Neurol 2009;8(8):724-730

51 Weber R, Minnerup J, Nordmeyer $\mathrm{H}$, et al. REVASK investigators. Thrombectomy in posterior circulation stroke: differences in procedures and outcome compared to anterior circulation stroke in the prospective multicentre REVASK registry. Eur J Neurol 2019;26(2):299-305
52 Kaesmacher J, Chaloulos-Iakovidis P, Panos L, et al. Clinical effect of successful reperfusion in patients presenting with NIHSS < 8: data from the BEYOND-SWIFT registry. J Neurol 2019;266(3):598-608

53 Singer OC, Berkefeld J, Nolte $\mathrm{CH}$, et al. ENDOSTROKE Study Group. Mechanical recanalization in basilar artery occlusion: the ENDOSTROKE study. Ann Neurol 2015;77(3):415-424

54 Premat K, Bartolini B, Baronnet-Chauvet F, et al. Single-center experience using the 3MAX reperfusion catheter for the treatment of acute ischemic stroke with distal arterial occlusions. Clin Neuroradiol 2018;28(4):553-562

55 Mistry EA, Mistry AM, Nakawah MO, et al. Mechanical thrombectomy outcomes with and without intravenous thrombolysis in stroke patients: a meta-analysis. Stroke 2017;48(9):2450-2456

56 Kaesmacher J, Mordasini P, Arnold M, et al. Direct mechanical thrombectomy in tPA-ineligible and -eligible patients versus the bridging approach: a meta-analysis. J Neurointerv Surg 2019;11(1):20-27

57 Delgado F, Oteros R, Jimenez-Gomez E. Bravo Rey I, Bautista MD, Valverde Moyano R. Half bolus dose of intravenous abciximab is safe and effective in the setting of acute stroke endovascular treatment. J Neurointerv Surg 2019;11(2):147-152

58 Heck DV, Brown MD. Carotid stenting and intracranial thrombectomy for treatment of acute stroke due to tandem occlusions with aggressive antiplatelet therapy may be associated with a high incidence of intracranial hemorrhage. J Neurointerv Surg 2015;7(3):170-175

59 Ernst M, Butscheid F, Fiehler J, et al. Glycoprotein IIb/IIIa inhibitor bridging and subsequent endovascular therapy in vertebrobasilar occlusion in 120 patients. Clin Neuroradiol 2016;26(2):169-175

60 Lavine SD, Cockroft K, Hoh B, et al. Training guidelines for endovascular ischemic stroke intervention: an international multi-society consensus document. AJNR Am J Neuroradiol 2016;37(4):E31-E34

61 Training guidelines for endovascular ischemic stroke intervention: an international multi-society consensus document. J Neurointerv Surg 2016;8(10):989-991

62 Day AL, Siddiqui AH, Meyers PM, et al. Training standards in neuroendovascular surgery: program accreditation and practitioner certification. Stroke 2017;48(8):2318-2325 